Supporting Information

\title{
Pore-spanning plasma membranes derived from giant plasma membrane vesicles
}

Nikolas K. Teiwes,,$^{\dagger}$ Ingo Mey, ${ }^{\dagger}$ Phila C. Baumann, ${ }^{\dagger}$ Lena Strieker, ${ }^{\dagger}$ Ulla Unkelbach, ${ }^{\dagger}$ Claudia Steinem ${ }^{\dagger, s}, *$

$\dagger$ Georg-August Universität, Institut für Organische und Biomolekulare Chemie, Tammannstr. 2, 37077 Göttingen, Germany

${ }^{\S}$ Max Planck Institute for Dynamics and Self-Organization, Am Fassberg 17, 37077 Göttingen, Germany

* Corresponding author: csteine@gwdg.de

1. Phase contrast images of a GPMV derived from PFA/DTT and calmidazolium treatment

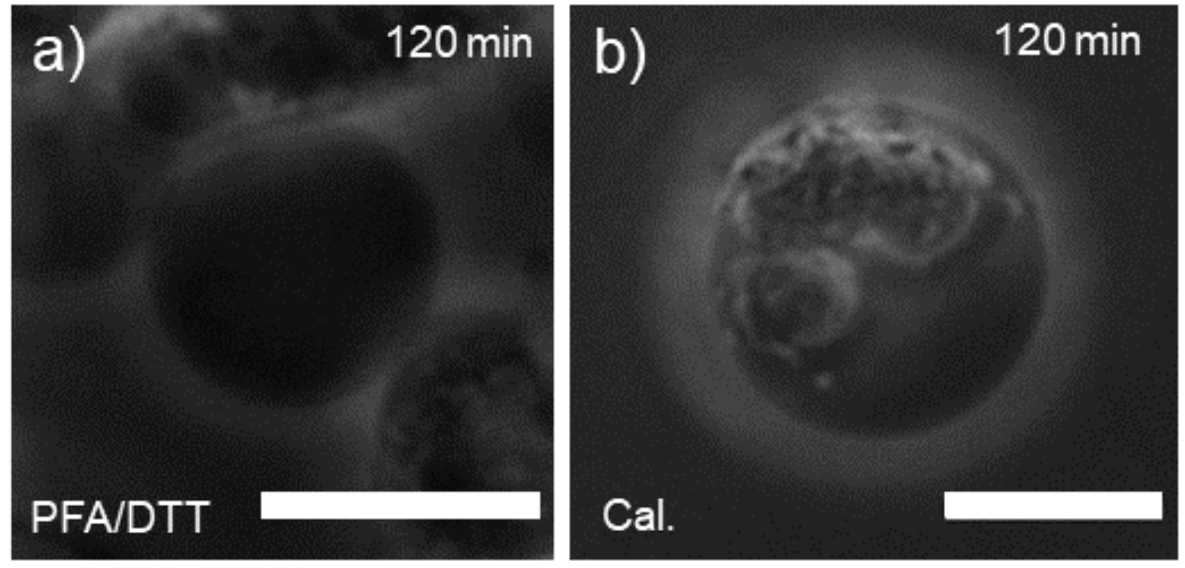

Figure S1. Phase contrast images of a GPMV obtained from a) PFA/DTT treatment and b) calmidazolium treatment after $120 \mathrm{~min}$. Scale bars: $20 \mu \mathrm{m}$.

\section{Visualization of a disordered and ordered phase in phase-separated GPMVs}
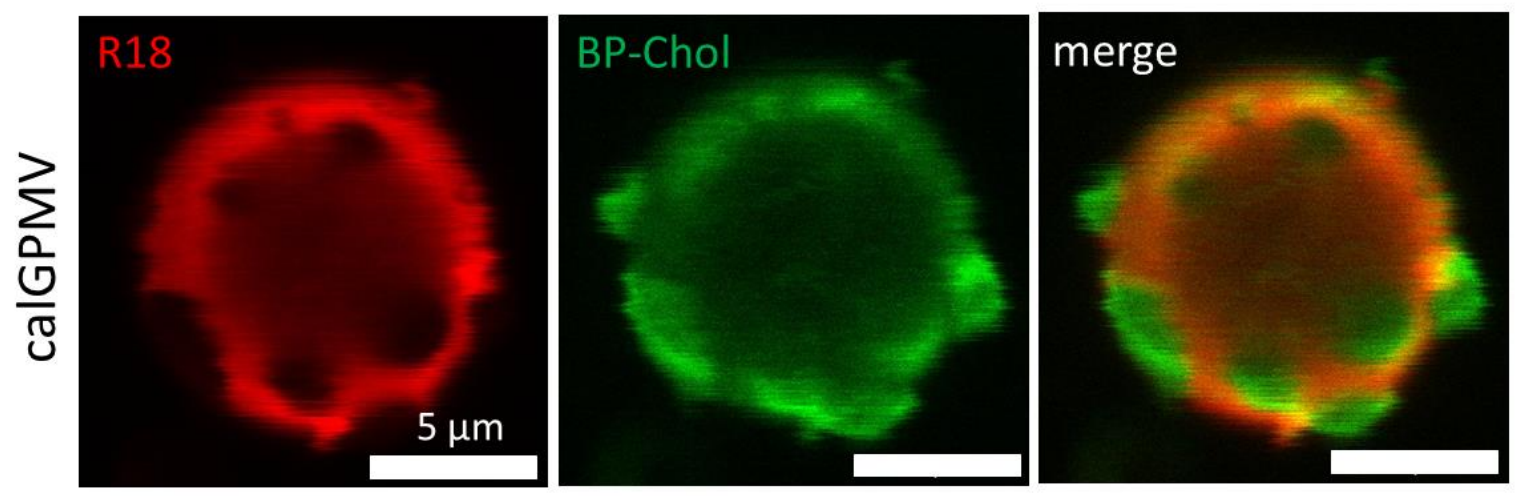

Figure S2. Fluorescence micrographs of a GMPV obtained by treatment with calmidazolium. R18 (red) labels the disordered phase, while Bodipy-cholesterol (green) partitions preferentially in the more ordered phase, where R18 is excluded. 


\section{Visualization of an F-actin network within GPMVs as a function of vesiculation agent}

HEK-293 cells, prelabeled with R18, were transfected with LifeAct-GFP (LA) (ibidi GmbH, Gräfelfing, Germany) and vesiculation agents were added as described. Fluorescence micrographs of R18 and LifeAct-GFP were taken (CLSM Microscope, Olympus, Plan-Apochromat 63×/1.0 $\left.\lambda_{\mathrm{ex}}^{\mathrm{R} 18}=561 \mathrm{~nm}, \lambda \lambda_{\mathrm{ex}}^{\mathrm{LA}}=488 \mathrm{~nm}, \lambda_{\mathrm{em}}^{\mathrm{R} 18}=580-735 \mathrm{~nm}, \lambda_{\mathrm{em}}^{\mathrm{LA}}=495-555 \mathrm{~nm}\right) . \quad$ Fluorescence images were analyzed with ImageJ (2.0.0-rc-69/1.53g). Details of data analysis are described in Figure S3. Within the straightened image the intensity in $x$-direction was averaged over the $y$-direction (Figure $\mathrm{S} 3 \mathrm{a}, \mathrm{b})$. The maximum peak intensities of the R18 fluorescence intensity profiles were used to determine the position of the membrane and to normalize the intensity. The distance $x$ is converted from pts to $\mu \mathrm{m}$ and the maximum R18 intensity is set to $x=0 \mu \mathrm{m}$ (dashed lines in Figure S3c). The resulting profile for the green channel (LA) was plotted for every GPMV with a thickened line $\left(\left(x \mid I_{\mathrm{LA}}^{\mathrm{norm}}\right)(0.1285 \mu \mathrm{m} \mid 0.0175\right.$ a.u. $\left.)\right)$ and a transparency < $1 / N(\mathrm{GPMV}) \%$ to ensure correct color coding of overlapping curves. The overlaps were recolored in a heat map, which is depicted in Figure S3c.

a)
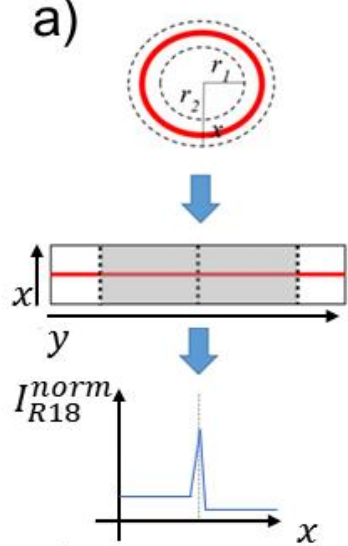

c)

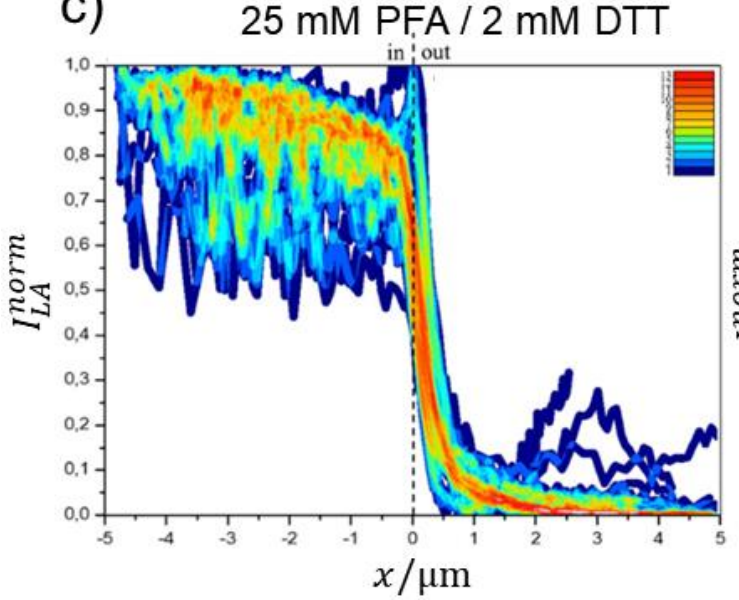

b)

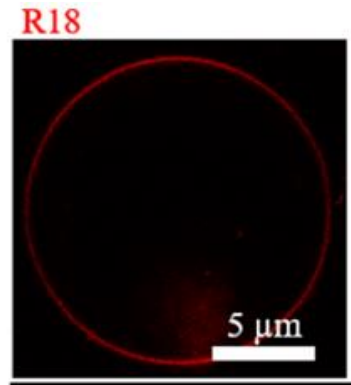

LifeAct

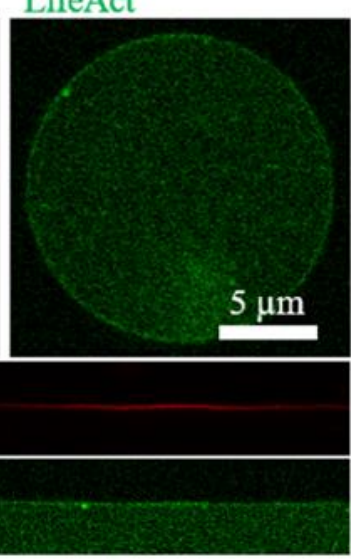

$10 \mu \mathrm{M}$ Calmidazolium

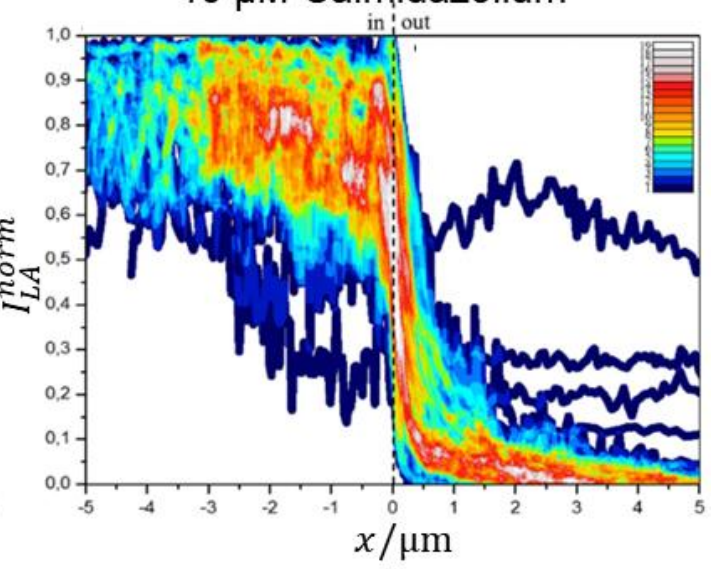

Figure S3. Fluorescence micrographs of GPMVs labeled with the membrane dye R18 and LifeAct-GFP. a) Schematic of the procedure to generate the line profiles shown in c). A donut-shaped 200 pts thick ROI $\left(x=r_{2}-r_{1}\right)$ was placed around the R18 labeled membrane of the GPMV to produce the intensity profile along $x$. A line scan orthogonal ( $x$-direction) to the membrane is used to average along the $y$-axis. b) Fluorescence micrographs of R18 and LifeAct-GFP of a single GPMV and after the procedure described in a). c) Intensity profiles obtained for LifeAct-GFP for GPMVs obtained after PFA/DTT treatment $(n=27)$ and calmidazolium $(n=49)$. All intensity profiles are shown in a heat map indicating that in case of PFA/DTT no peak at the membrane is observed, while GPMVs obtained with calmidazolium show a peak at about $250 \mathrm{~nm}$ to the inside from the membrane center (highlighted as a dashed line). 


\section{4. $\mathrm{SiO}_{2}$ oxygen plasma activation}

Table S1. Variation of oxygen plasma surface activation for supported plasma membrane bilayers (SPMB) and porespanning plasma membranes (PSPM). Spreading occurred directly after addition of the GPMVs in buffer solution and at room temperature. The portion (f-PSPM) is calculated for all patches by taking the total number f-PSPMs divided by the total number of pores within all patch areas.

\begin{tabular}{|c|c|c|c|c|c|c|}
\hline $\begin{array}{l}\text { System under } \\
\text { investigation }\end{array}$ & $\begin{array}{c}p\left(\mathrm{O}_{2}\right) / \\
\text { mbar }\end{array}$ & $t_{\mathrm{act}} / \mathrm{s}$ & $P^{\text {gen }} / \%$ & $N($ patch $)$ & $n$ (pore) & $\begin{array}{c}\text { Fraction (f-PSPM) / } \\
\%\end{array}$ \\
\hline SPMB & 0.2 & 3 & 10 & 299 & - & \\
\hline PSPM & 0.2 & 5 & 10 & \multirow[t]{4}{*}{104} & 745 & 18 \\
\hline PSPM & 0.2 & 3 & 10 & & 614 & 28 \\
\hline PSPM & 0.2 & 3 & 5 & & 231 & 36 \\
\hline PSPM & 0.2 & 3 & 3 & & 216 & 43 \\
\hline $\begin{array}{l}\text { GPMV (no } \\
\text { spreading) }\end{array}$ & 0.2 & 3 & 2 & \multicolumn{3}{|c|}{ only adhesion } \\
\hline $\begin{array}{l}\text { GPMV (no } \\
\text { spreading) }\end{array}$ & 0.2 & 3 & 1 & \multicolumn{3}{|c|}{ only adhesion } \\
\hline
\end{tabular}

\section{Adhesion and spreading of a GPMV}
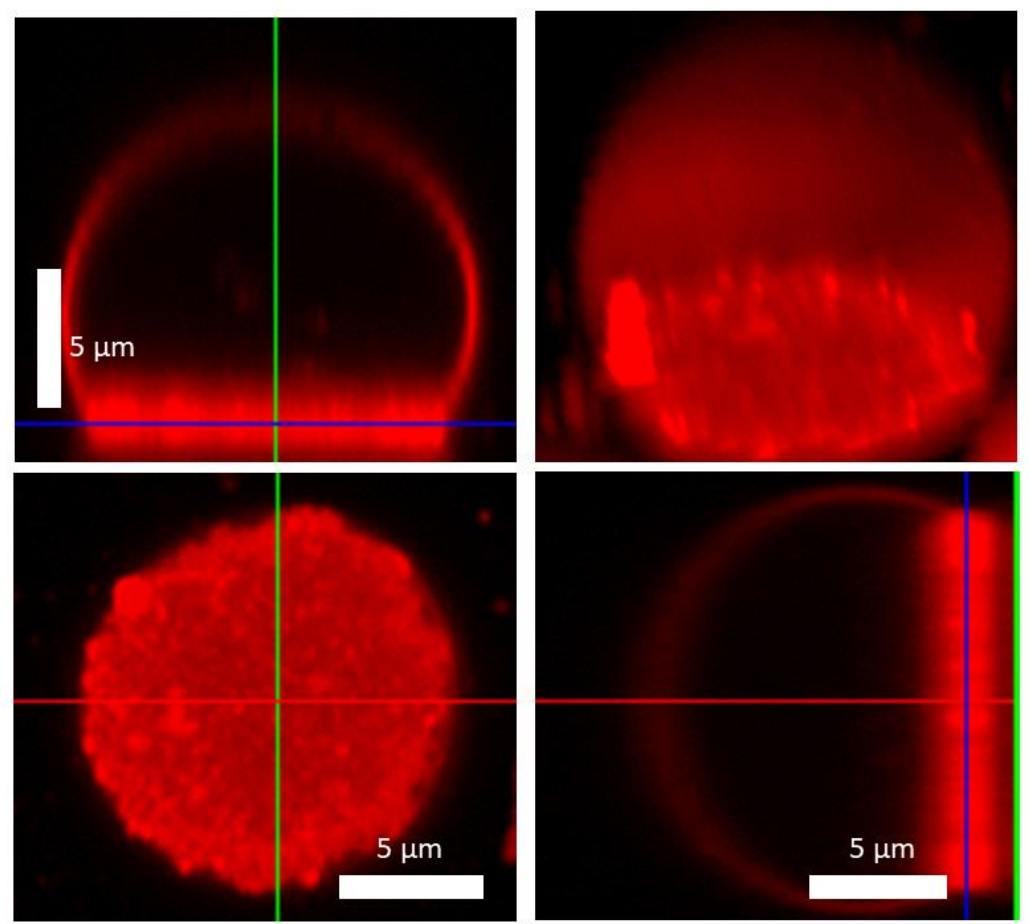

Figure S4. Fluorescence micrographs of an adhered GMPV on an activated silicon dioxide surface. 

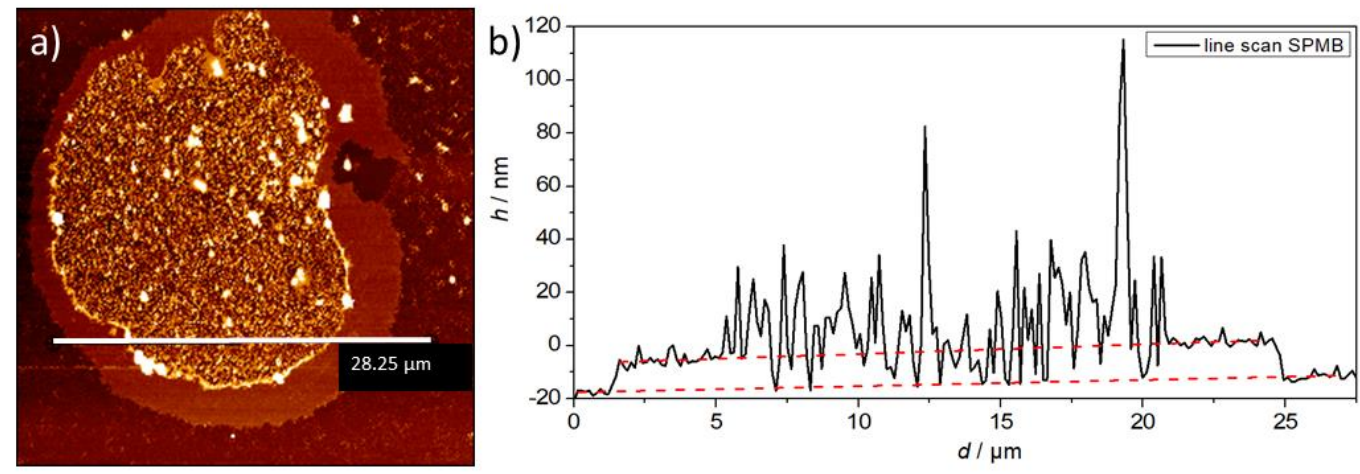

Figure S5. a) Atomic force micrograph of the planar supported plasma membrane patch shown in Figure 5c with b) the corresponding averaged line scan along the line shown in a).

\section{Fluorescence recovery after photobleaching (FRAP) experiments}

a)
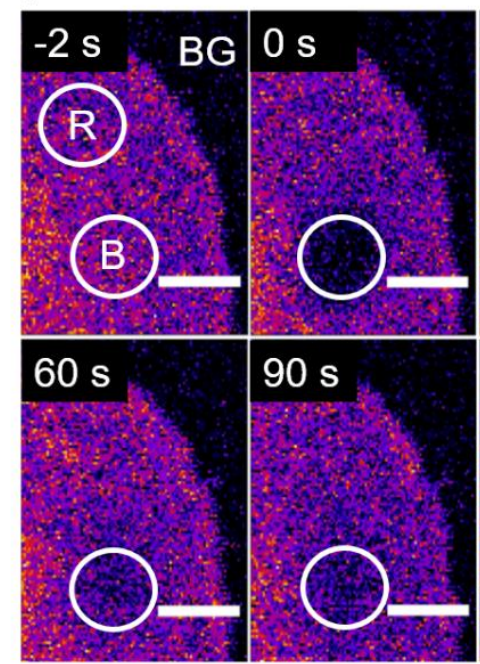

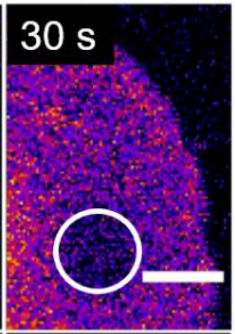

b)

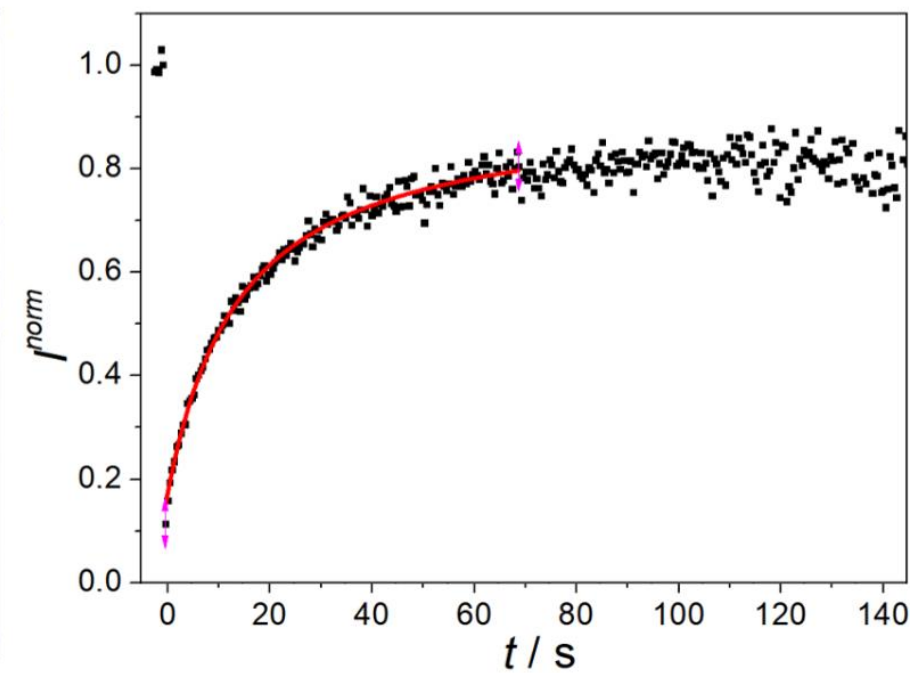

Figure S6. FRAP experiment on a supported plasma membrane produced by spreading of a calmidazolium induced GPMV on a $\mathrm{SiO}_{2}$ substrate a) False-colored fluorescence images at different time points of a FRAP experiment indicating the bleached region of interest $(\mathrm{ROI})(\mathrm{B} ; r=2.5 \mu \mathrm{m})$, the reference ROI (R) and the background (BG). Scale bars: $5 \mu \mathrm{m}$. b) Normalized FRAP curve (black squares) with $I_{\text {norm }}(t)=\frac{I_{\mathrm{B}}(t)-I_{\mathrm{BG}}}{I_{\mathrm{R}}(t)-I_{\mathrm{BG}}}$. The equation of Soumpasis: ${ }^{1} f(t)=A e^{-\frac{\tau_{D}}{2 t}}\left[I_{0}\left(\frac{\tau_{\mathrm{D}}}{2 t}\right)+\right.$ $\left.I_{1}\left(\frac{\tau_{\mathrm{D}}}{2 t}\right)\right]$ was used to analyze the FRAP curve with $\tau_{\mathrm{D}}=7.4 \pm 0.4 \mathrm{~s} ; A=1.36 \pm 0.03 \mathrm{~s} ; I_{0}=-0.43 \pm 0.03 \mathrm{~s}$ (red curve). 
Table S2. Diffusion coefficients $D=r^{2} / 4 \tau_{\mathrm{D}}$ and mobile fractions $M=\frac{I_{\text {norm }}(150 s)-I_{\text {norm }}(0 s)}{I_{\text {norm }}(-2 s)-I_{\text {norm }}(0 s)}$ of R18 within cells, PFA/DTT (pd) or calmidazolium (cal) treated GPMVs and supported plasma membrane bilayers (SPMB).

\begin{tabular}{|l|c|c|c|}
\hline \multicolumn{1}{|c|}{ Membrane origin } & $D / \mu \mathrm{m}^{2} / \mathrm{s}$ & $M / \%$ & $n$ \\
\hline Cell (top) & $1.3 \pm 0.5$ & $77 \pm 11$ & 21 \\
\hline Cell (bottom) & $0.6 \pm 0.4$ & $58 \pm 17$ & 19 \\
\hline pdGPMV & $4.7 \pm 3.4$ & $94 \pm 8$ & 19 \\
\hline calGPMV & $1.6 \pm 1.4$ & $77 \pm 19$ & 18 \\
\hline SPMB from pdGPMV & $1.0 \pm 0.8$ & $82 \pm 12$ & 17 \\
\hline SPMB from calGPMV & $0.4 \pm 0.1$ & $75 \pm 14$ & 16 \\
\hline
\end{tabular}

\section{SEM images of porous substrates}

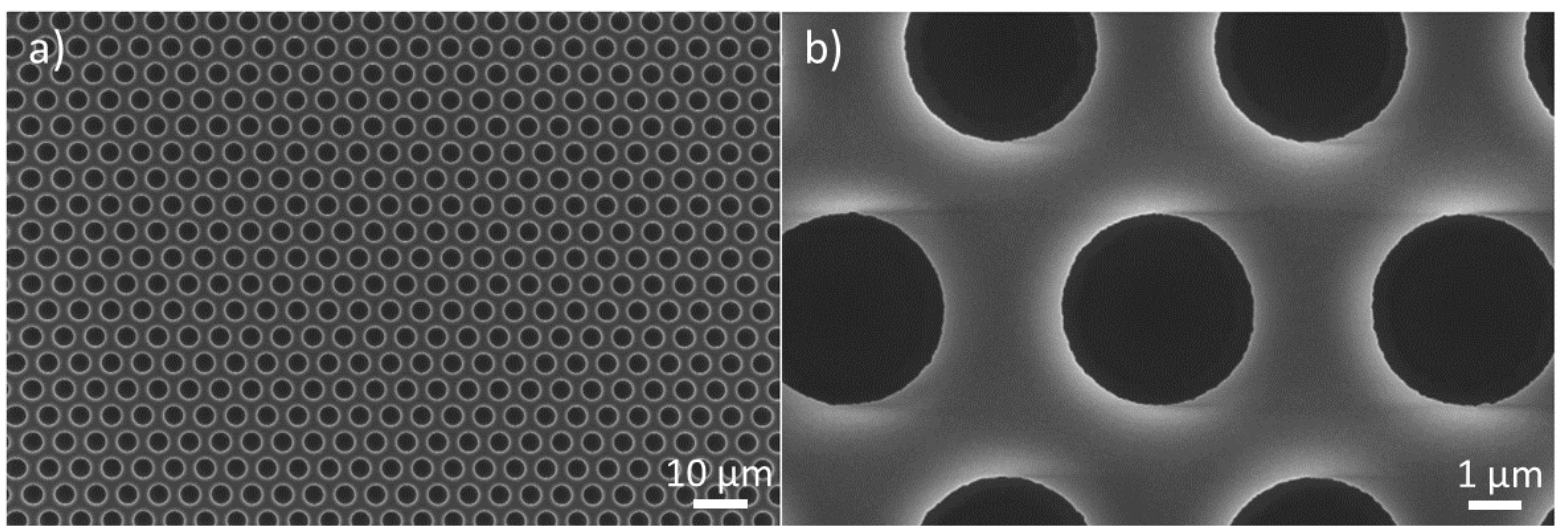

Figure S7. Scanning electron micrographs of the porous substrates $\left(\mathrm{SiO}_{2}\right.$-layer thickness $\left.>50 \mu \mathrm{m}\right)$ used in this study; a) overview, b) zoom in. A regular hexagonal pore array is visible with pore diameters of $3.5 \mu \mathrm{m}$.

\section{Movies}
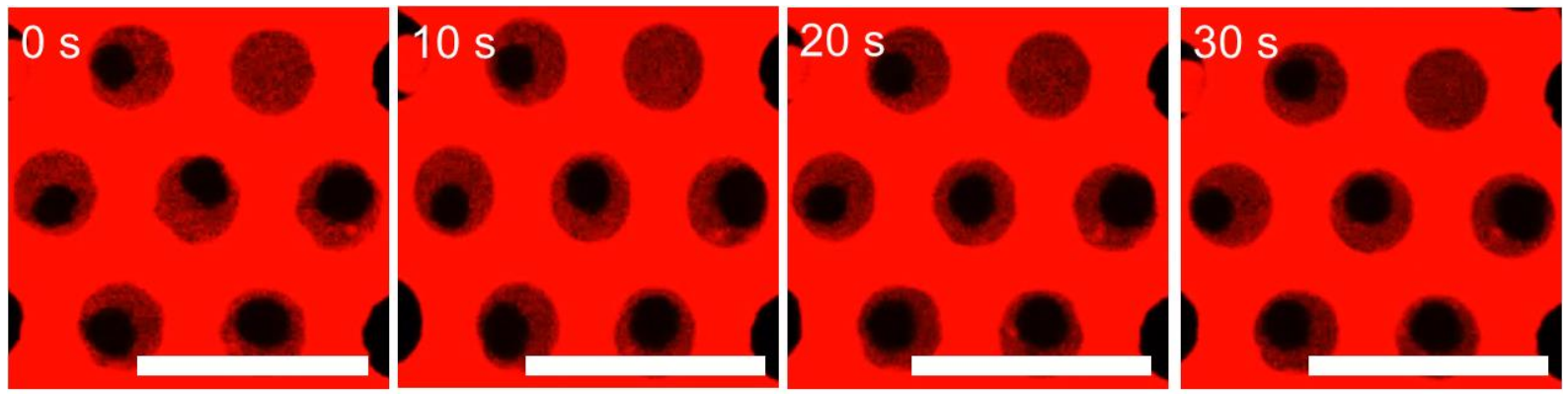

Movie S1. Ordered domain movement on f-PSPMs. Time lapse series of movie S1. Movie frame time: $6 \mathrm{fps}$, image acquisition time: $2.5 \mathrm{fps}$, Scale bars: $10 \mu \mathrm{m}$. 

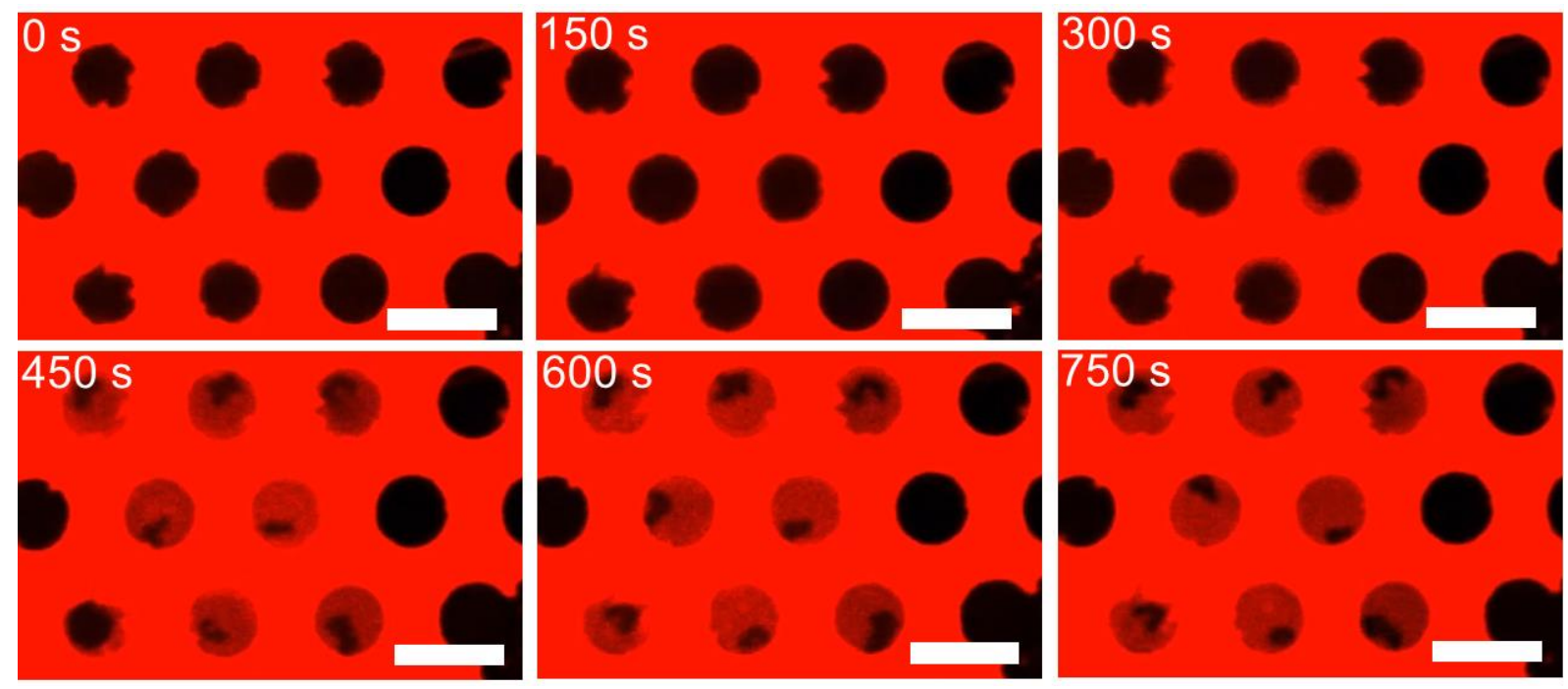

Movie S2. M $\beta C D$-cholesterol depletion of ordered domains on PSPMs. Time lapse series of movie S2. Movie frame time: $6 \mathrm{fps}$, image acquisition time $0.1 \mathrm{fps}$. Scale bars: $5 \mu \mathrm{m}$.

\section{References}

(1) Soumpasis, D. M. Theoretical analysis of fluorescence photobleaching recovery experiments. Biophys. J. 1983, 1983, 95-97. 